PREPARED FOR THE U.S. DEPARTMENT OF ENERGY, UNDER CONTRACT DE-AC02-76CH03073

PPPL-4003

PPPL-4003

UC-70

Aneutronic Fusion in a Degenerate Plasma

by

S. Son and N.J. Fisch

September 2004

N/M|

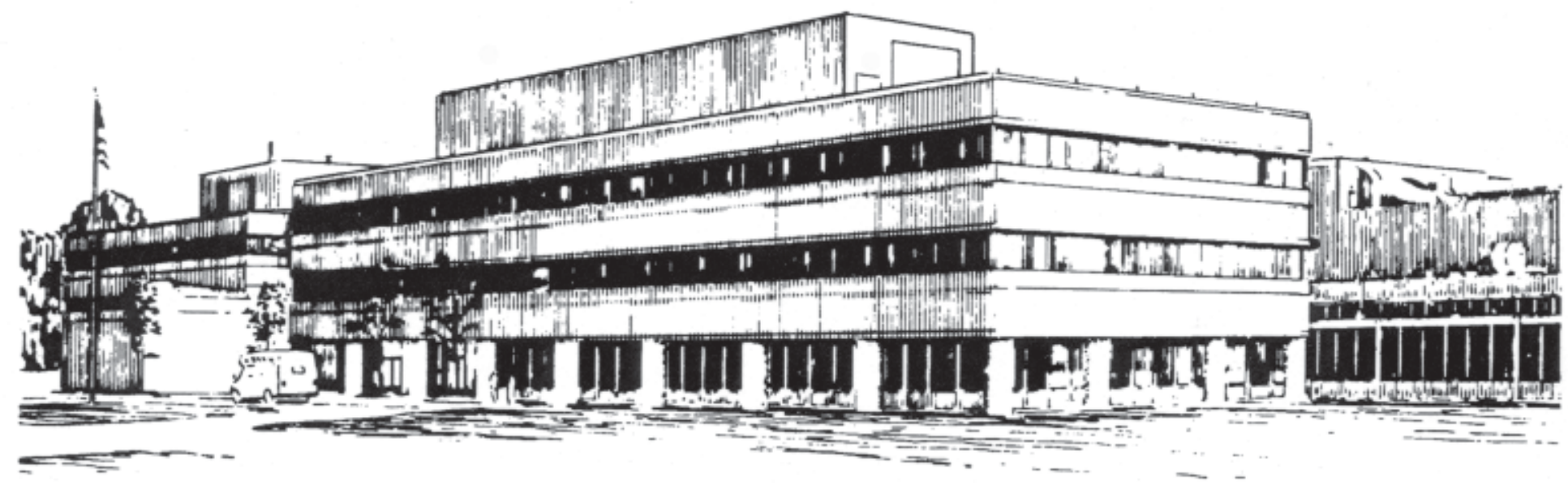

PRINCETON PLASMA PHYSICS LABORATORY PRINCETON UNIVERSITY, PRINCETON, NEW JERSEY 


\section{PPPL Reports Disclaimer}

This report was prepared as an account of work sponsored by an agency of the United States Government. Neither the United States Government nor any agency thereof, nor any of their employees, makes any warranty, express or implied, or assumes any legal liability or responsibility for the accuracy, completeness, or usefulness of any information, apparatus, product, or process disclosed, or represents that its use would not infringe privately owned rights. Reference herein to any specific commercial product, process, or service by trade name, trademark, manufacturer, or otherwise, does not necessarily constitute or imply its endorsement, recommendation, or favoring by the United States Government or any agency thereof. The views and opinions of authors expressed herein do not necessarily state or reflect those of the United States Government or any agency thereof.

\section{Availability}

This report is posted on the U.S. Department of Energy's Princeton Plasma Physics Laboratory Publications and Reports web site in Fiscal Year 2004. The home page for PPPL Reports and Publications is: http://www.pppl.gov/pub_report/

DOE and DOE Contractors can obtain copies of this report from:

U.S. Department of Energy

Office of Scientific and Technical Information

DOE Technical Information Services (DTIS)

P.O. Box 62

Oak Ridge, TN 37831

Telephone: (865) 576-8401

Fax: (865) 576-5728

Email: reports@adonis.osti.gov

This report is available to the general public from:

National Technical Information Service

U.S. Department of Commerce

5285 Port Royal Road

Springfield, VA 22161

Telephone: $1-800-553-6847$ or

(703) $605-6000$

Fax: (703) 321-8547

Internet: http://www.ntis.gov/ordering.htm 


\title{
Aneutronic Fusion in a Degenerate Plasma
}

\author{
S. Son and N.J. Fisch \\ Princeton Plasma Physics Laboratory, Princeton University
}

\begin{abstract}
In a Fermi-degenerate plasma, the electronic stopping of a slow ion is smaller than that given by the classical formula, because some transitions between the electron states are forbidden. The bremsstrahlung losses are then smaller, so that the nuclear burning of an aneutronic fuel is more efficient. Consequently, there occurs a parameter regime in which self-burning is possible. Practical obstacles in this regime that must be overcome before net energy can be realized include the compression of the fuel to an ultra dense state and the creation of a hot spot. +
\end{abstract}

Key words:

aneutronic, fusion, degeneracy, stopping, bremsstrahlung, proton, boron, helium, deuterium

PACS: 52.27.Gr, 52.27.-z, 52.25.Mq

\section{Introduction}

A fusion reaction in which no neutrons are produced is safer and cleaner than D-T fusion reactor. $P+B^{11} \rightarrow 3 \alpha(2.7 \mathrm{MeV})$ and $D+H e^{3} \rightarrow p(14.7 \mathrm{MeV})+$ $\alpha(3.6 \mathrm{MeV})$ are the most promising reaction for this purpose. However, the cross-section for these fuels is appreciable only when the ion-temperature $T_{i}$ exceeds $100 \mathrm{KeV}$ for P-B-11, and $50 \mathrm{KeV}$ for D-He-3 [1,2]. P-B-11 is the cleanest, but it needs high temperature for burning. D-He-3 requires only a moderate temperature, but it has two disadvantages. One is the production of the neutrons from the D-D and D-T reactions. This can be partially overcome with a high-He-3 and low-D fuel mixture, which then must be burned above $100 \mathrm{KeV}$ [3]. The other drawback is the scarcity of He-3 on Earth [4].

For P-B-11, Dawson [3] pointed out that the bremsstrahlung power at temperature of $200 \mathrm{KeV}$ is greater than the fusion power, which makes self-burning

Email addresses: sson@pppl.gov, fisch@pppl.gov (S. Son and N.J. Fisch). 
unlikely. To avoid the bremsstrahlung losses, the electron temperature $T_{e}$ must be much lower than the ion temperature $T_{i}$, but not too low because the fusion byproducts should be preferentially stopped by the ions $[3,5]$. In view of this consideration, the electron temperature must be in narrow range around $100 \mathrm{keV}$ to retain the possibility of self-burning [3,5-7].

For inertial confinement fusion using P-B-11, there have been several theoretical attempts to generate a detonation wave [5,8-10]. Martinez-Val and Eliezer [8] showed that compressed fuel can be burned by an expanding ion fusionburning wave preceded by an electron-conduction heat detonation wave. A large gap between the electron temperature $T_{e} \cong 80 \mathrm{KeV}$ and the ion temperature $T_{i} \cong 200 \mathrm{KeV}$ might then be achievable. Leon [9] suggested that the bremsstrahlung, the stopping power of an alpha particle, and the ion-electron collision rate are are all reduced due to the electron degeneracy, facilitating the detonation wave. However, the feasibility of self-burning even with this temperature differential still remains unclear [5]. We show here that, without consideration of the practicality, for a particular parameter regime, Fermi degeneracy plays an important role in reducing the alpha particle stopping, ion-electron collisions and the bremsstrahlung, so that self-burning is possible. The optimal regimes are characterized by an electron temperature much lower than the $80 \mathrm{KeV}$ suggested by Eliezer and Martinez-Val [5,8].

The feasibility of ICF in D-He-3 [4] is greater if the fusion reactivity is higher. In D-He-3 fusion, a $14 \mathrm{MeV}$ proton transfers its energy mainly to the electrons, and so $T_{e}$ is the same or higher than $T_{i}$. Because of the bremsstrahlung losses, the concentration of $\mathrm{D}$ has a minimum value, under which a burning is impossible [3]. By reducing this minimum value, we can achieve the minimum number of neutrons. Honda [4] pointed out that, due to the nuclear elastic scattering, there will be more energy transfer to the ions from a $14 \mathrm{MeV}$ proton, which though still smaller than to the electrons, improves the fusion reactivity. We show that, due to the degeneracy, the proton can be stopped mainly by He-3 through the nuclear elastic scattering, and the fuel might be burned in low $T_{e}$ and high $T_{i}$. Accessing a regime in which $T_{i} \gg T_{e}$ is always useful for achieving controlled fusion. For example, in magnetically confined fusion, the low electron temperature reduces the requirement on confining plasma pressure, and the reactivity can be improved by channeling the alpha energy in the D-T reaction [12]. In a tokamak, this might be accomplished through rf waves [11]. Here, the regime $T_{i} \gg T_{e}$ affords the possibility of achieving ignition altogether in aneutronic fuel.

The paper is organized as follows. In Sec. II, the stopping power formula in a electron degenerate plasma is presented. In Sec. III. the regime of self-burning is identified using the formula in Sec. II. In Sec. IV, the $\rho R$ equation for fuel burning is solved to find the appropriate pellet dimension and the constraint on the laser or ion beam power. In Sec. V, we discuss the implications and 
and limitations of these results. In Sec. VI, we summarize our main results.

\section{$2 \quad$ Electronic Stopping Power}

The electronic stopping power in an electron degenerate metal has been intensively studied theoretically [13,15-23] and experimentally $[22,24-29]$. In a fully degenerate plasma, when the velocity of an ion is smaller than the electron Fermi-velocity, the electronic stopping power becomes almost independent of the density and proportional to the ion velocity $[13,15,17]$, i.e.,

$$
c \frac{d E}{d t}=C(\chi) \frac{8}{3 \pi} \frac{m^{2} Z^{2} e^{4}}{\mu \hbar^{3}} E,
$$

where $\mu$ is the ion mass, $E$ is the ion energy, $m$ is the electron mass, $\chi^{2}=$ $e^{2} / \pi \hbar v_{F}, v_{F}$ is the Fermi velocity, and $C(\chi) \cong \frac{1}{2}\left[\log \left(1+\frac{1}{\chi^{2}}\right)-\frac{1}{1+\chi^{2}}\right]$ [16]. The above formula is valid if $v \ll v_{F}$ and $r_{s} \ll 1$, where $v$ is the ion velocity, and $r_{s}=\frac{m e^{2}}{\hbar}\left(\frac{3}{4 \pi n_{e}}\right)^{1 / 3}[13,15,17]$. The collisions occur between the ion and the fastest electrons rather than, as in a weakly-coupled hot plasma, between the ion and the thermal electrons. The collisional cross-section decreases as $1 / v_{F}^{4}$. This strong dependence of the cross-section on $v_{F}$ just suffices to cancel the effect of the greater electron density, the greater energy loss per collision, and the great relative velocity of the colliding particles. The stopping frequency then is independent of the electron density. The ion-electron collision frequency is

$$
\nu_{e, i}=3.47 \times 10^{13}\left(Z^{2} / \mu\right) \frac{1}{\sec }
$$

where $\mu$ is the nucleus mass in the unit of the proton mass, and $C(\chi) \cong 2$ when $n \cong 10^{28}\left(1 / \mathrm{cm}^{3}\right)$. For further details, see Appendix A.

\section{$3 \quad$ Regimes of Self-burning}

\section{$3.1 P-B-11$}

When $T_{e}<T_{i}$, the ion kinetic energy is drained into the electrons. The rate of the energy drain from the ions to the electrons is given as $P_{i, e}\left(\mathrm{eV} / \mathrm{cm}^{3} \mathrm{sec}\right)=$ $\frac{3}{2} \nu_{i, e} n_{i} T_{i}$ with $\nu_{i, e}$ given from Eq. $(2)$, where $n_{i}\left(T_{i}\right)$ is the total density (temperature) of the ions, and $T_{i} \gg T_{e}$ is assumed. The rate of the fusion energy 
production is $P_{f}\left(\mathrm{eV} / \mathrm{cm}^{3} \mathrm{sec}\right)=n_{1} n_{2}\langle\sigma v\rangle \triangle E$, where $n_{1}$ and $n_{2}$ is the density of the fusing pair, $\triangle E$ is the energy produced per fusion, and $\langle\sigma v\rangle$ is the Maxwellian average or the so-called "reaction activity" [1]. We now consider $\mathrm{P}-\mathrm{B}-11$ fuel. The ratio $P_{i, e} / P_{f}$ is given as

$$
\frac{P_{i, e}}{P_{f}}=\frac{(25 / 11 \epsilon+1)(5 \epsilon+1)}{\epsilon} \frac{3.47 \times 10^{13}}{n_{e}\langle\sigma v\rangle} \frac{3 T_{i} / 2}{8.7 \mathrm{MeV}},
$$

where $\epsilon=n_{B} / n_{p}$. For the fuel to be burned, firstly, $P_{i, e} / P_{f}<1$ must be satisfied, and secondly, the fusion product must be stopped mainly by the ions not by the electrons. Note that Eq.(3), as a function of $\epsilon$, has a minimum when $\epsilon \cong 0.3$. With $\epsilon=0.3$ and $T_{i}=200 \mathrm{KeV}$, we note that $P_{i, e} / P_{f}=1$ when the electron density is $n_{0}=6.69 \times 10^{28}\left(1 / \mathrm{cm}^{3}\right)$. Thus $n_{e}>n_{0}$ satisfies the first requirement. We used the recent reaction activity data from [30]: $\langle\sigma v\rangle=2.5 \times 10^{-16}\left(\mathrm{~cm}^{3} / \mathrm{sec}\right)$, which is less than the activity given by the old data by $37.5 \%[5]$.

For the second, with $\epsilon=0.3$, the ion stopping frequency of the alpha particle is $\sum_{j} \nu_{\alpha, j}(E)=7.32 \times 10^{13}\left(n_{e} / n_{0}\right)\left(E_{0} / E\right)^{3 / 2}(1 / \mathrm{sec})$, where $E_{0}=2.7 \mathrm{MeV}$, the Coulomb logarithm is $\Lambda=5$, and we used the classical formula : $\nu_{i, j} \cong$ $9.0 \times 10^{-8}\left(\frac{n_{j} Z_{j}^{2}}{\mu_{j}} \Lambda_{i, j}\right) \sqrt{\mu_{i}} \frac{1}{E_{i}^{3 / 2}}$. The fraction of the energy transfer from the alpha particle to the ions is

$$
\int_{0}^{E_{0}} d E \frac{\sum_{j} \nu_{\alpha, j}(E)}{\nu_{i, e}+\sum_{j} \nu_{\alpha, j}(E)} .
$$

When $n_{e}=n_{0}, 85 \%$ of the alpha particle energy goes to ions; for $n_{e}=2 n_{0}$, the fraction is $92 \%$. Therefore, the second requirement is satisfied automatically when $n_{e}>n_{0}$.

The electron temperature is determined from the balance between the energy input from the ions and the losses from the bremsstrahlung:

$$
P_{i, e}\left(T_{e}, T_{i}\right)=P_{B}\left(T_{e}\right)
$$

For $n_{e}=2 n_{0}$ and $\epsilon=0.3, P_{i, e}=9.3 \times 10^{47}\left(\mathrm{eV} / \mathrm{cm}^{3} \mathrm{sec}\right)$. Using the classical bremsstrahlung formula $[3,5,34]$,

$$
P_{B}\left(\frac{\mathrm{eV}}{\mathrm{cm}^{3} \mathrm{sec}}\right)=9.3 \times 10^{-14} n_{e} T^{1 / 2}\left(\Sigma_{i} n_{i} Z_{i}^{2}\right)\left(1+\frac{2 T_{e}}{m_{e} c^{2}}\right)
$$

where $T_{e}$ in the unit of $\mathrm{eV}$. We obtain $T_{e} \cong 27 \mathrm{KeV}$ from Eq.(5). The above analysis shows that, in principle, P-B-11 can be burned with $T_{i}=200 \mathrm{KeV}$ 
and $T_{e}=27 \mathrm{KeV}$, with the optimized fuel concentration $\epsilon=0.3$ overcoming the bremsstrahlung losses. The density of the system is slightly more than $3.8 \times 10^{5}\left(\mathrm{~g} / \mathrm{cm}^{3}\right)$, and the Fermi energy is $95 \mathrm{KeV}$. Since $T_{e}<E_{F}$, the electrons are still degenerate.

\subsection{D-He-3}

In D-He-3 ICF, the electron temperature can generally be no less than the ion temperature, since a $14 \mathrm{MeV}$ proton is mainly stopped by electrons. For example, if $n_{D} / n_{H e}=0.1$ to assure a low neutron level, then $P_{B} / P_{f}$ is larger than 1 at all temperatures [3], and a self-sustaining burn is not possible. However, nuclear-elastic-collisions(NEC) do channel energy from a $14 \mathrm{MeV}$ proton to He-3 [4,31], improving the chance for a self-sustaining burn. Nonetheless, since most of the energy still goes from the proton to the electrons, the electron temperature still cannot be much lower than the ion temperature. Thus, it appears, at first sight, necessary to increase $n_{D} / n_{H e}$ for burning. We show, however, that, in an ultra dense $\rho=10^{5}\left(\mathrm{~g} / \mathrm{cm}^{3}\right)$ plasma, the NEC can transfer the proton energy mainly to He-3, and so the electron temperature might be lower than the ion temperature as in P-B-11 case, thereby, achieving the self-sustaining burn condition.

If Coulomb stopping of the proton by the ions is ignored, the energy loss of the proton by the ions is given by

$$
\frac{d E}{d t}=-\sigma_{N}(E) v(E) f(E)
$$

where $\sigma_{N}(E)$ is NEC cross-section, $v$ is the proton velocity, and $f$ is the fraction of the proton energy loss per a NEC. The fraction of the energy deposition to the electrons is

$$
r_{e}=\int_{0}^{E_{0}} \frac{\nu_{i, e} E}{\nu_{i, e} E+\sigma_{N}(E) v(E) f(E)} d E,
$$

where $E_{0}=14.7 \mathrm{MeV}, \nu_{i, e}=3.47 \times 10^{13}(1 / \mathrm{sec})$ from Eq.(2). Using NEC data from [38], roughly, $r_{e} \cong 1 /\left(1+n_{H e} /\left(3.47 * 10^{28}\right)\right)$. For $\rho=3 \times 10^{5}\left(\mathrm{~g} / \mathrm{cm}^{3}\right)$ and for $n_{D} / n_{H e}=0.1,35 \%$ goes to electrons. The alpha particle also transfers less than 10 percent of its energy to the electrons. Overall, $70 \%$ of energy goes to the ions from the fusion product. For $n_{d} / n_{H e}=0.1, T_{i}=70 \mathrm{KeV}$ and $\rho$ as given above, we note that $P_{i, e}=2.94 \times 10^{47}\left(\mathrm{eV} / \mathrm{cm}^{3} \mathrm{sec}\right)$, and $P_{f}=5.79 \times$ $10^{47}\left(\mathrm{eV} / \mathrm{cm}^{3} \mathrm{sec}\right)$, where we used the reactivity data: $\langle\sigma v\rangle=10^{-16} \mathrm{~cm}^{3} / \mathrm{sec}$ from [1], and that $P_{B}=2.6 \times 10^{45} \sqrt{T_{e}}\left(\mathrm{eV} / \mathrm{cm}^{3} \mathrm{sec}\right)$, where $T_{e}$ is in $\mathrm{eV}$ and assumed to be non-relativistic. We note also that $0.7 P_{f} / P_{i, e}=1.38>1$, and 
so the fuel burns. By balancing $P_{i, e}+0.3 P_{f}=P_{B}$, we find $T_{e}=32 \mathrm{KeV}$. The plasma is still partially degenerate since $E_{F}=90 \mathrm{KeV}$.

\section{Reactor Prospects}

To find the pellet dimension and total power, we solve the $\rho R$ equation ( see e.g. [32]) in P-B-11 with $\epsilon=0.3$ and $\rho=3.8 \times 10^{5}\left(\mathrm{~g} / \mathrm{cm}^{3}\right)$ :

$$
\frac{d x}{d t} \cong 1.2 \times 10^{13}(0.7+x)
$$

where $x$ is the ratio of the deuterium density to the initial helium density; $x=0.3$ at $t=0$, and $x=0$ at total burn-up. The solution is $x(0.7+x) \cong$ $0.3 e^{-1.2 \times 10^{13} t}$. For the total burn-up, the confinement time $t_{c}=R / C_{s}$ must be larger than $10^{-13}$ sec, where $C_{s}$ is the sound wave velocity, and $R$ is the pellet

dimension. Assuming $C_{s} \cong \sqrt{n E_{F} / \rho}$, then $R$ must be larger than $10^{-4} \mathrm{~cm}$. The electron degeneracy energy is $3 \times 10^{9} \mathrm{~J} / \mathrm{g}$. As an example, for $R=10^{-3} \mathrm{~cm}$, by putting $P_{\text {in }}=4.78 \mathrm{MJ}$, we get $P_{\text {out }}=88 \mathrm{MJ}$, and so $G=P_{\text {out }} / P_{\text {in }}=18.31$. For D-He-3 with $n_{D} / n_{H e}=0.1$ and $\rho=3 \times 10^{5} \mathrm{~g} / \mathrm{cm}^{3}$, the pellet dimension and the energy input characteristic is almost the same as with P-B-11 analysis. The gain $G$ is 15 .

The feasibility as a reactor for either of these fuels is low because the gain is smaller than 20, and more than 200 is usually required [36]. We note that the gain can be as large as 1000 in D-T fuel [32]. The creation of a hot spot for the fast ignition [33] might be a way of improving the gain substantially. This might be done by using a small D-T pellet inside the aneutronic fuel or a fission-fusion hybrid concept [4]. Because of the ultra dense condition, a difficult practical requirement for the uniformity of the laser or particle beam in compression must be met.

\section{Discussion}

As shown in Sec. III, the radiation losses can be overcome sufficiently for selfsustained burning. Rider [37] pointed that the fusion power of an aneutronic plasma is substantially smaller than the minimum recirculating power to maintain the non-equilibrium condition ( high $T_{i}$ and low $T_{e}$ ), which diminishes the prospect for utilizing aneutronic fuel. However, his derivation is under the assumption that the two-body effects are proportional to $\int d^{3} \mathbf{x}|n(x)|^{2}$. In an ultra dense plasma, we showed the stopping power is not proportional to the 
electron density, breaking that assumption, and thus avoiding the negative conclusion by Rider.

The practicality as a reactor is likely small, as discussed in Sec. IV, unless the gain can be made larger. In this respect, note that certain assumptions made here might be too pessimistic. One is that we assumed total electron degeneracy in the calculation of $\nu_{i, e}$. In a partially degenerate plasma, $\nu_{i, e}$ has the tendency to decrease as a function of $T_{e}[39,43,41,42]$, but the detailed result of the stopping power in a partially degenerate plasma $[44,43,45]$ has not been incorporated into our calculation.

To see why, in a partially degenerate plasma, the slowing down might be smaller yet, consider a plasma in which electrons can be assumed to be classical. When $v \ll v_{t e}$, where $v\left(v_{t e}\right)$ is the velocity of the ion (the thermal electron), the ion energy loss comes solely from collisions of the ion with the electrons with $\left|v_{e}\right|<v$. For an isotropic velocity distribution, electrons with $\left|v_{e}\right|>v$ do not drag the ion because of a well-known cancellation(e.g. [35]).

On the other hand, if $v \ll v_{F}$, and the electrons are completely degenerate, then the drag on the ion comes mainly from the electrons with $\left|v_{e}\right| \cong v_{F}$. The force from electrons with $\left|v_{e}\right| \cong v_{F}$ does not cancel in contrast to the classical limit. This is because, due to the lack of the asymmetry of the electron-hole transition probability [13], the drag force of electrons on a ion is not exactly an inverse-square law. It depends on the direction relative to the ion-velocity. The cancellation, however, occurs only for inverse-square forces. Electrons with $\left|v_{e}\right|<v_{F}-v$ do not drag the ion because these electrons do not collide with the ion due to the lack of available holes.

Consider now a case when $v \ll v_{t e} \ll v_{F}$, where $v_{t e}=\sqrt{2 T_{e} / m_{e}}$. The electrons with $\left|v_{e}\right|<v$ still do not drag the ions, because no hole is available. The drag by the electrons with $\left|v_{e}\right| \cong v_{F}$ is greatly reduced compared to the case of the complete degeneracy, since the transition probability asymmetry is not very sharp, but has instead the scale of $v_{t e}$. Its effect can be roughly estimated, and it might imply that $\nu_{i, e}$ must be reduced by $O\left(v_{t e} / v_{F}\right)$ compared to Eq.(3).

When $T \cong E_{F}$, mainly electrons with $\left|v_{e}\right|<v$ contribute to the stopping. The stopping frequency is then proportional to $\nu_{e, i} \cong \nu_{e, i}^{c} e^{-\mu} /\left(1+e^{-\mu}\right)^{2}$, where $\mu$ is the chemical potential, and $\nu_{e, i}^{c}$ is the classical ion-electron collision frequency with $T_{e} \cong E_{F}$.

The above rough considerations seem to imply, if speculative, that if $v \ll v_{t e} \ll$ $v_{F}, \nu_{i, e}$ is reduced further as a function of $T_{e}$ around $m_{e} v^{2} / 2<T_{e} \leq E_{F}$. This is especially true for P-B-11 because $v_{t i} / v_{t e} \cong 0.1$ where $v_{t i}=\sqrt{2 T_{i} / m_{i}}$ with $T_{e}=10 \mathrm{KeV}$ and $T_{i}=200 \mathrm{KeV}$. In D-He-3, the $14 \mathrm{MeV}$ proton velocity is too large to have a such a separation. But in P-B-11, we might speculate 
that there will be big reduction of the stopping frequency for an appropriate electron temperature.

Secondly, the bremsstrahlung is also reduced. When $E_{F} \gg T_{e}$, not all electrons collide with the ions, since many of the electron-hole transitions are forbidden. The estimate [40], using the classical derivation of the bremsstrahlung [34], shows that the total loss will be reduced by $O\left(\left(T / E_{F}\right)^{3 / 2}\right)$ from the classical formula. If the bremsstrahlung is reduced too much so that the electrons begin to heat up, we can put some high-Z impurity to the fuel so that we can fine tune the bremsstrahlung to balance with the ion-electron energy transfer at the optimal electron temperature.

Thirdly, at such a high density as $n_{e} \cong 10^{29}\left(1 / \mathrm{cm}^{3}\right)$, $\hbar \omega_{p e}$ becomes $10 \mathrm{KeV}$, where $\omega_{p e}=\sqrt{4 \pi n e^{2} / m_{e}}$ is the plasma frequency, and a significant fraction of the energy radiated will be re-absorbed, given the fact that $T_{e}$ is a few tens of $\mathrm{KeV}$. The Compton heating of the electrons also turns out to be significant $[5]$.

The above considerations tell us that the severe condition imposed for selfburning in Sec. III can be eased by the further reduction in $\nu_{i, e}$, the bremsstrahlung losses and the re-absorption. However, this is all quite speculating; an estimation of how much it will help remains to be seen. In particular, the bremsstrahlung and the stopping power should be taken into account in the full context of the partial degeneracy. We can adopt the bremsstrahlung losses from [40] and a more exact estimation of $\nu_{i, e}$ from $[44,43,45]$.

As a warning, we note the following: In the stopping power estimation (Sec. 2), we assume that $n_{e}=10^{28} \mathrm{~cm}^{-3}$ with $T_{e}=0$, but for $n_{e}=10^{29} \mathrm{~cm}^{-3}$, we note a $10 \%$ increase in the electron stopping compared with Eq.(2). We also note that the relativistic effect is also should be taken into account in the calculation of the stopping power and the bremsstrahlung, because the Fermi energy is $20 \%$ of the electron mass energy. We estimate that, in the bremsstrahlung, the partial degeneracy is much more important effect than the relativistic one [40]. But, in the stopping power reduction, the relativistic effect might be as important as the partial degeneracy [46].

Therefore, we propose that the full time evolution of the fuel burning should be obtained with the relativistic effect, the partial degeneracy, the local field correction and the other effects mentioned taken into account. While this is beyond the scope of the present manuscript, it is clear to the extent that these effects tend to reduce the coupling of the electrons, and it will be even easier to maintain disparate ion and electron temperature and hence greater activity. 


\section{Summary}

In this paper, we identified a possible ignition regime for P-B-11 and D-He-3, in which $\rho>10^{5}(\mathrm{~g} / \mathrm{cm}), T_{i} \cong 100 \mathrm{KeV}$, and $T_{e}=30 \mathrm{KeV}$. The degeneracy of the electrons reduces the stopping power and the bremsstrahlung losses, which facilitates self-sustained burning. It is mainly the reduction in the stopping power of the electrons that enables such a large differential between ion and electron temperature. While the power requirements suggest that this regime is still impractical for inertial confinement fusion, the regime may be practical should the present assumptions turn out to be wrong concerning the electron stopping in partially degenerate plasma, the Compton heating, the reduction of the bremsstrahlung, relativistic effect, or the re-absorption of the radiation. Some arguments are given suggesting that these assumptions, in fact, may overstate the stopping by electrons.

The Authors thank R. Kulsrud, G. Hammett and S. Cohen for useful discussions. This work was supported by the U.S. DOE under contract AC0276CH0-3073

\section{Appendix A: Stopping Power in a Degenerate Plasma [15]}

The total field generated by a test particle, which travels with velocity $\mathbf{v}$ at the position at $\mathbf{r}_{\text {test }}(t)=\mathbf{r}_{0}+\mathbf{v} t$, is

$$
E_{\mathbf{k}, \omega}^{t o t}=\frac{E_{\mathbf{k}, \omega}^{t e s t}}{D(\mathbf{k}, \omega)}
$$

where $\mathbf{E}_{\mathbf{k}, \omega}^{\text {test }}=(2 \pi q) e^{-i \mathbf{k} \cdot \mathbf{r}_{0}} \delta(\omega-\mathbf{k} \cdot \mathbf{v})$ is the Fourier transform of $\mathbf{E}^{\text {test }}(\mathbf{r})=$ $q\left(\mathbf{r}-\mathbf{r}_{\text {test }}(t)\right) /\left|\mathbf{r}-\mathbf{r}_{\text {test }}(t)\right|^{3}$. The field generated from the background particles is

$$
\begin{aligned}
\mathbf{E}^{p o l}\left(\mathbf{r}_{\text {test }}, t\right) & =\int \mathbf{E}^{p o l}(\mathbf{k}, \omega) e^{i \mathbf{k} \cdot \mathbf{r}_{\text {test }}-i \omega t} \frac{d^{3} \mathbf{k}}{(2 \pi)^{3}} \frac{d \omega}{(2 \pi)} \\
& =q \int \epsilon_{k}\left[\frac{1}{D(\mathbf{k}, \mathbf{k} \cdot \mathbf{v})}-1\right] \frac{d^{3} \mathbf{k}}{(2 \pi)^{3}},
\end{aligned}
$$

which becomes [15]

$$
\mathbf{E}^{p o l}\left(\mathbf{r}_{t e s t}, t\right)=-4 \pi q \int\left[\frac{\mathbf{k} \cdot \mathbf{v}}{k^{2} v} \frac{\operatorname{Im} D(\mathbf{k}, \mathbf{k} \cdot \mathbf{v})}{|D(\mathbf{k}, \mathbf{k} \cdot \mathbf{v})|^{2}}\right] \frac{d^{3} \mathbf{k}}{(2 \pi)^{3}}
$$


The stopping power $\frac{d K}{d l}=\left|q \mathbf{E}^{\text {pol }}\right|$ is the energy loss of the particle per unit length by the drag, where $K$ is the kinetic energy of the ion. From Eq.(11), $\frac{d K}{d l}$ is given as

$$
\frac{d K}{d l}=\frac{q^{2}}{2 \pi^{2}} \int\left[\frac{\mathbf{k} \cdot \mathbf{v}}{k^{2} v} \frac{\operatorname{Im} D(\mathbf{k}, \mathbf{k} \cdot \mathbf{v})}{|D(\mathbf{k}, \mathbf{k} \cdot \mathbf{v})|^{2}}\right] d^{3} \mathbf{k} .
$$

Now we consider the drag on the test ion by the degenerate electrons. The dielectric function $D^{l}$ in a completely degenerate plasma is given as

$$
D^{l}=1+\frac{3 \omega_{p e}^{2}}{k^{2} v_{F}^{2}} f,
$$

where $f$ is

$$
f(u, z)=\left(\begin{array}{c}
\frac{1}{2}+\frac{1}{8 z}\left(1-(z-u)^{2}\right) \log \left(\frac{z-u+1}{z-u-1}\right) \\
+\frac{1}{8 z}\left(1-(z+u)^{2}\right) \log \left(\frac{z+u+1}{z+u-1}\right)
\end{array}\right),
$$

where $z=\frac{k}{2 k_{F}}, u=\frac{\omega+i \gamma \hbar}{k v_{F}}$, and $v_{F}\left(k_{F}\right)$ is the Fermi velocity(wave vector). We note that $f$ has the real part $f_{r}$ and the imaginary part $f_{i}$. For a very small $\gamma, f_{r}$ are given as

$$
\begin{gathered}
f_{r}=\left(\begin{array}{c}
\frac{1}{2}+\frac{1}{8 z}\left(1-(z-u)^{2}\right) \log \left(\left|\frac{z-u+1}{z-u-1}\right|\right) \\
+\frac{1}{8 z}\left(1-(z+u)^{2}\right) \log \left(\left|\frac{z+u+1}{z+u-1}\right|\right.
\end{array}\right) \\
f_{i}=\left(\begin{array}{c}
\frac{\pi}{2} u,(|z+u|<1) \\
\frac{\pi}{8 z}\left(1-(z-u)^{2}\right),(|z-u|<1<|z+u|) \\
0,(|z-u|>1)
\end{array}\right) .
\end{gathered}
$$

Using Eqs.(13) and (15), Eq.(12) becomes

$$
\frac{d K}{d l}=\frac{4 \pi Z^{2} e^{4}}{m_{e} v^{2}} n_{e} L
$$

where $L$ is

$$
L=\frac{6}{\pi} \int_{0}^{\frac{v}{v_{F}}} u d u \int_{0}^{\infty} z^{3} d z \frac{f_{i}(u, z)}{\left(z^{2}+\chi^{2} f_{r}(u, z)\right)^{2}+\chi^{4} f_{i}(u, z)^{2}},
$$


where $\chi^{2}=\frac{e^{2}}{\pi \hbar v_{F}}$. We now consider the case when $\frac{v}{v_{F}} \ll 1$. From Eq.(15), $f_{i}$ and $f_{r}$ is given as

$$
\begin{gathered}
f_{r}(u, z) \cong f_{r}(0, z) \\
f_{i}(u, z) \cong\left(\begin{array}{r}
\frac{\pi}{2} u,(z<1) \\
0,(z>1)
\end{array}\right) .
\end{gathered}
$$

$\frac{d K}{d l}$ in Eq.(12) then becomes

$$
\frac{d K}{d l}=\frac{4 Z^{2} e^{4} m_{e}^{2}}{3 \pi \hbar^{3}} v C_{1}(\chi)
$$

where $C_{1}$ is defined as

$$
C_{1}(\chi)=\int_{0}^{1} \frac{z^{3} d z}{\left(z^{2}+\chi^{2} f_{r}(0, z)\right)^{2}} .
$$

Eqs.(20), (19) shows that when $v / v_{F} \ll 1$, the energy loss is proportional to the ion velocity, and almost independent of the electron density. If we put $f_{r}=f_{r}(0,0)=1$, and assume $\chi^{2} \ll 1, C_{1} \cong-\log (\chi)$ which is obtained by Fermi [13]. $\nu_{e, i}$ then can be obtained from $\nu_{e, i}=\left(\frac{d K}{d l}\right) / K v$

\section{References}

[1] G.H. Miley and H. Towner and N. Ivich, Fusion cross-sections and reactivities, Report C00-2218-17 (1974).

[2] J.R. Mcnally, Nucl. Fusion 11, 187 (1971).

[3] J. Dawson, Fusion, Volume 2, ( Academic Press, New York, 1981 ).

[4] T. Honda, Y. Nakao, Y. Honda, and K. Kudo, Nuclear Fusion 31, 851 (1991).

[5] S. Eliezer and J.M. Martinez-Val, Laser and Particle Beams 16, 581 (1998).

[6] C. Scheffel, R.J. Stening, H. Hora, R. Hopfl, J.M. Marinez-Val, S. Eliezer, G. Kasotakis, M. Piera, and E. Sarris, Laser and Particle Beams 15, 565 (1997).

[7] Y. Setsuhara, H. Azechi, N. Miyanaga, H. Furukawa, R. Ishizawa, K. Nishihara, M. Katayama, A. Nishiguchi, K.Mima and S. Nakai", Laser and Particle Beams 8, 609 (1990). 
[8] J.M. Martinez-Val and S. Eliezer and M. Piera and G. Velarde, Physics Letters A 216, 142 (1996).

[9] P.T. Leon, S. Eliezer, J.M. Martinez-Val, and M. Piera, Physics Letters A 289, 135 (2001).

[10] B. Levush and S. Cuperman, Nucl. Fusion 22, 1519 (1982).

[11] N.J. Fisch and J.M. Rax, Physical Review Letters 69, 612 (1992).

[12] N.J. Fisch and M.C. Herrmann, Nucl. Fusion 35,1753 (1995).

[13] E. Fermi and E. Teller, Physical Reviews 72, 399 (1947)

[14] J. Meyer-Ter-Vehn, Nuclear Fusion 22, 561 (1982).

[15] J. Lindhard and K. Dan Vidensk. Selsk, Mat. Fys. Medd. 28, No. 8, (1954).

[16] I. Nagy, J. Laszlo and J. Giber, Nucl. Inst. Meth. Phys. B 27, 276 (1987).

[17] I. Nagy, A. Arnau and P.M. Echenique, Physical Review B 40, 11983 (1989).

[18] I. Nagy, Physical Review B 62, 5270 (2000).

[19] I. Nagy, A. Arnau and P.M. Enchenique, Physical Review A 43, 6038 (1991).

[20] J.P. Biersack, Nucl. Inst. Meth. phys. B 166, 19 (2000)

[21] W.K. Chu and D. Powers, Physical Review 187, 478 (1969).

[22] C.D. Moak and M.D. Brown, Physical Review 149, 244 (1966).

[23] C.Deutsch and C. Maynard, Physical Review A 340, 3209 (1989).

[24] M. Fama, J.C. Eckardt, G.H. Lantschner, N.R. Arista, Phys. Rev. Lett. 85, 4486 (2000).

[25] S.K. Allison, Rev. Mod. Phys. 25, 779 (1953).

[26] F.W. Meyer, C.C. Havener and P.A.Z. Vanemmichoven, Physical Review A 48, 4479 (1993).

[27] T. Kaneko, Physical Reivew A, 33, 1602 (1986).

[28] W. White and R.M. Mueller, Physical Review 187, 499 (1969).

[29] I. Gertner and M. Meron and B. Rosner, Physical Review A 18, 2022 (1978).

[30] W.M. Nevins and R. Swain, Nuclear Fusion 40, 865 (2000).

[31] Y. Nakao, T. Honda and K.Kudo, Nucl. Fusion 30, 143 (1990).

[32] M.D. Rosen, Phys. Plasmas 6, 1690 (1999).

[33] M. Tabak, J. Hammer, M.E. Glinsky, W.L. Kruer, S.C. Wilks, J. Woodworth, E.M. Campbell, M.D. Perry, and R.J. Mason, Phys. Plasmas 1, 1626 (1994). 
[34] J. Greene, ApJ 130, 693 (1959).

[35] B.A. Trubinikov, Review of Plasma physics, Volume 1, 105 ( Consultant Bureau, New York, 1965).

[36] R.E. Kidder, Nuclear Fusion 19, 223 (1979).

[37] T.H. Rider, Phys. Plasmas 4, 1039 (1997).

[38] S.T. Perkins and D.E. Cullen, Nucl. Sci. Eng 77, 20 (1981)

[39] I. Nagy, A. Arnau and P.M. Echenique, Physical Review A 43, 6038 (1994).

[40] S. Eliezer, P.T. Leon, J.M. Martinez-Val and D.V. Fisher, Laser and Particle Beams 21, 599 (2003).

[41] N.R. Arista, J. Phys. C: Solid State Phys. 18, 5127 (1985)

[42] S. Skupsky, Physical Review A, 16, 727 (1977).

[43] G. Maynard and C. Deutsch, Physical Review A 26, 665 (1982).

[44] G. Gouedard and C. Deutsch, J. Math. Phys. 19, 32 (1978).

[45] C. Deutsch and G. Maynard, J.Physique 46, 1113 (1985).

[46] B. Jancovici, Nuovo Cimento 25, 428 (1962). 


\section{External Distribution}

Plasma Research Laboratory, Australian National University, Australia

Professor I.R. Jones, Flinders University, Australia

Professor João Canalle, Instituto de Fisica DEQ/IF - UERJ, Brazil

Mr. Gerson O. Ludwig, Instituto Nacional de Pesquisas, Brazil

Dr. P.H. Sakanaka, Instituto Fisica, Brazil

The Librarian, Culham Laboratory, England

Mrs. S.A. Hutchinson, JET Library, England

Professor M.N. Bussac, Ecole Polytechnique, France

Librarian, Max-Planck-Institut für Plasmaphysik, Germany

Jolan Moldvai, Reports Library, Hungarian Academy of Sciences, Central Research Institute for Physics, Hungary

Dr. P. Kaw, Institute for Plasma Research, India

Ms. P.J. Pathak, Librarian, Institute for Plasma Research, India

Ms. Clelia De Palo, Associazione EURATOM-ENEA, Italy

Dr. G. Grosso, Instituto di Fisica del Plasma, Italy

Librarian, Naka Fusion Research Establishment, JAERI, Japan

Library, Laboratory for Complex Energy Processes, Institute for Advanced Study, Kyoto University, Japan

Research Information Center, National Institute for Fusion Science, Japan

Dr. O. Mitarai, Kyushu Tokai University, Japan

Dr. Jiangang Li, Institute of Plasma Physics, Chinese Academy of Sciences, People's Republic of China

Professor Yuping Huo, School of Physical Science and Technology, People's Republic of China

Library, Academia Sinica, Institute of Plasma Physics, People's Republic of China

Librarian, Institute of Physics, Chinese Academy of Sciences, People's Republic of China

Dr. S. Mirnov, TRINITI, Troitsk, Russian Federation, Russia

Dr. V.S. Strelkov, Kurchatov Institute, Russian Federation, Russia

Professor Peter Lukac, Katedra Fyziky Plazmy MFF UK, Mlynska dolina F-2, Komenskeho Univerzita, SK-842 15 Bratislava, Slovakia

Dr. G.S. Lee, Korea Basic Science Institute, South Korea

Institute for Plasma Research, University of Maryland, USA

Librarian, Fusion Energy Division, Oak Ridge National Laboratory, USA

Librarian, Institute of Fusion Studies, University of Texas, USA

Librarian, Magnetic Fusion Program, Lawrence Livermore National Laboratory, USA

Library, General Atomics, USA

Plasma Physics Group, Fusion Energy Research Program, University of California at San Diego, USA

Plasma Physics Library, Columbia University, USA

Alkesh Punjabi, Center for Fusion Research and Training, Hampton University, USA

Dr. W.M. Stacey, Fusion Research Center, Georgia Institute of Technology, USA

Dr. John Willis, U.S. Department of Energy, Office of Fusion Energy Sciences, USA

Mr. Paul H. Wright, Indianapolis, Indiana, USA 
The Princeton Plasma Physics Laboratory is operated by Princeton University under contract with the U.S. Department of Energy.

\author{
Information Services \\ Princeton Plasma Physics Laboratory \\ P.O. Box 451 \\ Princeton, NJ 08543
}

Phone: 609-243-2750

Fax: 609-243-2751

e-mail: pppl_info@pppl.gov

Internet Address: http://www.pppl.gov 